\title{
On the CP asymmetries in Majorana neutrino decays
}

\author{
Esteban Roulet ${ }^{1}$, Laura $_{\text {Covi }}{ }^{2}$ and Francesco Vissani ${ }^{3}$ \\ ${ }^{1}$ Depto. de Física Teórica, Universidad de Valencia, \\ E-46100, Burjasot, Valencia, Spain \\ ${ }^{2}$ School of Physics and Chemistry, Lancaster University \\ Lancaster LA1 $4 Y B$, United Kingdom \\ 3 Deutsches Elektronen Synchroton, DESY \\ 22603 Hamburg, Germany
}

\begin{abstract}
We study the $\mathrm{CP}$ asymmetries in lepton number violating two body scattering processes and show explicitly how they vanish, in agreement with unitarity constraints. We relate these cross section asymmetries to the $\mathrm{CP}$ decay rate asymmetries of the intermediate massive neutrinos and show how the inclusion of the Universe expansion via Boltzmann equations is the key ingredient to produce a non-vanishing asymmetry in spite of the unitarity constraint on the cross sections. We then show that the absorptive parts of both the one loop vertex and self energy corrections do contribute to the CP decay asymmetries.
\end{abstract}


To generate dynamically a cosmological baryon (or lepton) asymmetry, two crucial ingredients are the existence of $\mathrm{CP}$ violation and the required out of equilibrium condition (the third one being just the existence of $\mathrm{B}$ or $\mathrm{L}$ violating interactions). We want in this note to examine carefully these two issues with the aim of clarifying an existing debate in the literature. We will concentrate in the discussion of leptogenesis scenarios [1], in which a baryon asymmetry results after reprocessing an initial lepton asymmetry, whose origin is related to the existence of heavy Majorana neutrinos. However, our conclusions are general and apply to all scenarios based on out of equilibrium decays.

The CP asymmetries are usually computed by studying the decay rate asymmetries of an unstable heavy particle. This asymmetry arises in general through the interference of a tree level process with a one loop diagram containing an absorptive part. One such diagram is depicted in fig. 1a and gives the so-called 'vertex' contribution. The point in debate is whether the absorptive part of the 'self-energy' like diagrams (fig. 1b) also contributes to the partial decay asymmetries. The existence of this kind of contributions was noticed a few times [2]- [9], but they are still not always included (see e.g. refs. [10]-13]); furthermore, it has been argued in a recent paper [14] that the self energy contributions should cancel among themselves, and hence be irrelevant for the generation of a baryon (or lepton) asymmetry.

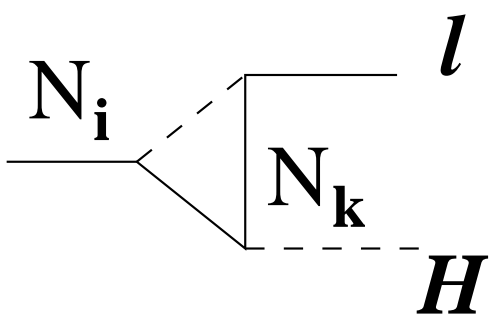

(a)

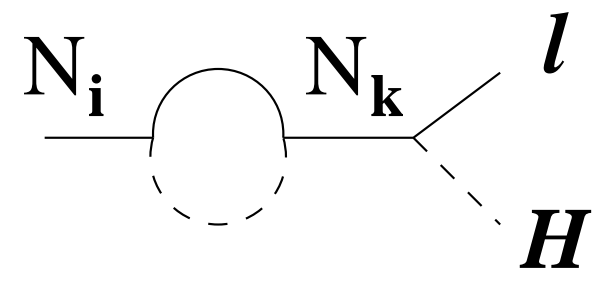

(b)

Figure 1: One loop diagram which interferes with the tree level amplitude to give a CP asymmetry in the decay of a heavy singlet neutrino $N$, coupled through a Yukawa interaction to the standard lepton and Higgs doublets.

The usual argument invoked to include these absorptive self-energy parts in the computation of the decay rates is that they cannot be reabsorbed 
in the wave function renormalisation of the heavy states without loosing the hermiticity of the Lagrangian, and hence they are physical. On the other side, in ref. 114 it was argued that the heavy states, being unstable, are not a good starting point for perturbative calculations. In this spirit, it was suggested to use cross section asymmetries involving stable asymptotic states, in which the heavy unstable particles appear only as virtual intermediate states. The following $\mathrm{CP}$ asymmetry was studied:

$$
\epsilon_{\sigma} \propto \sigma\left(\ell^{c} H^{*} \rightarrow \ell H\right)-\sigma\left(\ell H \rightarrow \ell^{c} H^{*}\right)
$$

(Here and in the following we will always assume that there is an implicit sum in the flavour of the initial and final leptons, and in the flavour of the intermediate heavy neutrinos.) After computing in detail all the selfenergy contributions to this quantity, a complete cancellation was found. It was then concluded that only the vertex diagrams could provide a nonvanishing contribution. The results in [14] indeed reproduce what one would obtain just including the vertex contributions to direct and inverse decay rate asymmetries.

However, unitarity considerations imply that $\epsilon_{\sigma}$ defined in eq. (11) is actually exactly zero at the one-loop level. In fact, unitarity implies that the probabilities for all possible transitions to and from a state $i$ should sum to one, i.e.

$$
\sum_{j}|M(i \rightarrow j)|^{2}=\sum_{j}|M(j \rightarrow i)|^{2}
$$

where the sum over $j$ includes all states and antistates. In the case of leptogenesis, one may take $j=1,2,3$ to describe $\ell_{j}$ and $j=4,5,6$ to describe $\ell_{j-3}^{c}$. Hence, writing for brevity only the leptonic fields, the asymmetry $\epsilon_{\sigma}$ will be proportional to

$$
\begin{aligned}
\epsilon_{\sigma} & \propto \sum_{i=4}^{6} \sum_{j=1}^{3}\left[\sigma\left(\ell_{i} \rightarrow \ell_{j}\right)-\sigma\left(\ell_{j} \rightarrow \ell_{i}\right)\right] \\
& =\sum_{i=1}^{6} \sum_{j=1}^{3}\left[\sigma\left(\ell_{i} \rightarrow \ell_{j}\right)-\sigma\left(\ell_{j} \rightarrow \ell_{i}\right)\right] \\
& =\sum_{i=1}^{6} \sum_{j=1}^{3}\left[\sigma\left(\ell_{j} \rightarrow \ell_{i}\right)-\sigma\left(\ell_{j} \rightarrow \ell_{i}\right)\right]=0
\end{aligned}
$$




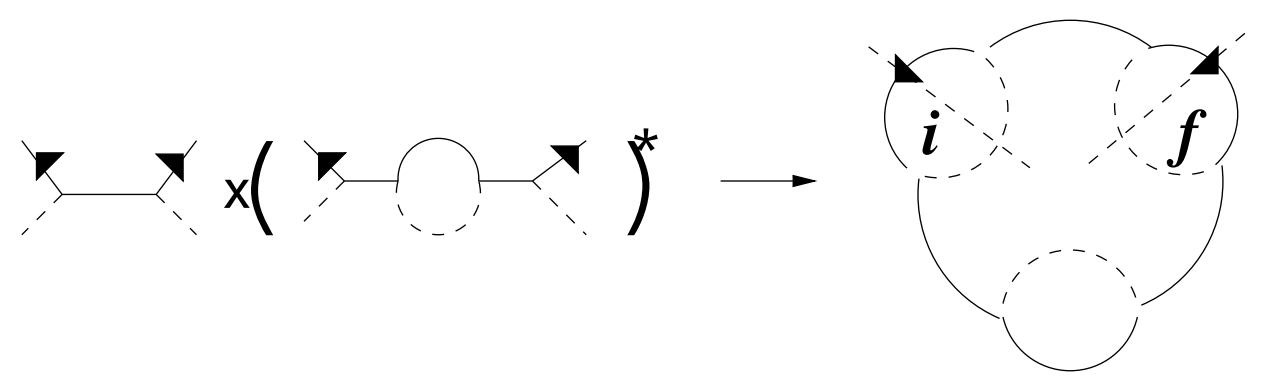

Figure 2: Pictorial representation with cut blobs of the interference term of the cross section $\sigma\left(\ell^{c} H^{*} \rightarrow \ell H\right)$. Higgs scalars are represented with dashed lines. Initial and final leptons with solid lines with arrows corresponding to the lepton number flow.

where we first added and subtracted the lepton conserving scattering processes and then used the unitarity relation in eq. (2) appliied to the $2 \rightarrow 2$ scatterings|.

The non-zero result obtained in ref. [14] is due to the omission of some diagrams: those associated to a further tree level process, in which $N$ is exchanged in the $u$-channel?.

There is a simple and instructive way to see pictorially how all the different diagrams cancel out, which we first illustrate by reproducing the cancellation of the self-energy parts found in ref. [14]. For this sake we will use the fact that the interference term in the cross section (the only one contributing to the asymmetries) is proportional to $\operatorname{Re}\left[\mathcal{M}_{0} \mathcal{M}_{1}^{*}\right]$, where $\mathcal{M}_{0(1)}$ is the tree (one loop) amplitude.

Therefore, one can pictorially represent the self-energy contributions to $\sigma\left(\ell^{c} H^{*} \rightarrow \ell H\right)$ as in figure 2, where the cut blobs are the initial $(i)$ and final $(f)$ states, while the remaining blob stands for the one-loop self-energy. This last is actually the sum of two contributions, one with a lepton and one with an antilepton.

\footnotetext{
${ }^{1}$ Beyond $O\left(\lambda^{6}\right)$, where $\lambda$ are the Yukawa couplings, final states with more than two particles will appear in the unitarity relation. These multiparticle states will also have to be included in the definition of $\epsilon_{\sigma}$.

${ }^{2}$ There are also one loop box diagrams, but they preserve lepton number (as can be seen by hypercharge conservation), hence they do not contribute to the asymmetry considered. Loop diagrams in the $u$-channel exchange are irrelevant at leading order since they have no absorptive parts.
} 


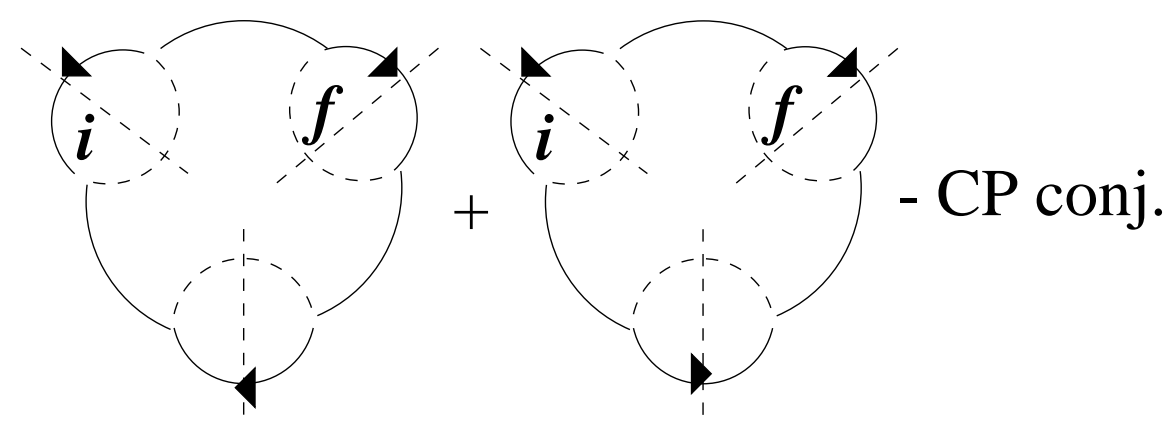

Figure 3: Pictorial representation of the self-energy contribution to the cross section asymmetry $\epsilon_{\sigma}$.

Now, in the computation of $\epsilon_{\sigma}$, only the absorptive part of the loop will contribute, and the Cutkoski rule then tells us that the self-energy blob should also be cut. Hence, putting explicitly the two contributions to the selfenergy and using the Cutkoski rule, the asymmetry $\epsilon_{\sigma}$ will be proportional to the diagrams in figure 3 .

In the first diagram, the lepton is running clockwise in two blobs and counterclockwise in the third blob, while in the second diagram only one lepton is running clockwise while the other two go in the reverse direction (drawing conventionally the leptonic lines towards the external part of the diagram). The CP-conjugate processes, which have to be subtracted to obtain the asymmetry, have just the arrows reversed, and hence under conjugation the two diagrams shown change into each other. This leads to a complete cancellation of the asymmetry.

Turning now to the vertex contributions, to see the cancellations we need to add the three contributions shown in figure 4 (including the tree level $u$ channel interfering with the one-loop self-energy diagram). In terms of cut diagrams, this can be expressed as in figure 5, where CP-conjugate stands for the same four diagrams with all the arrows reversed. Hence, the CPconjugate contribution will exactly cancel the four diagrams, since changing the directions of the arrows just exchanges among themselves the first and fourth diagrams, as well as the second and third ones. We then see explicitly that the absorptive part of the self-energies are also playing here a crucial role, enforcing the cancellation of the $\mathrm{CP}$ violation produced by the vertex diagrams. 

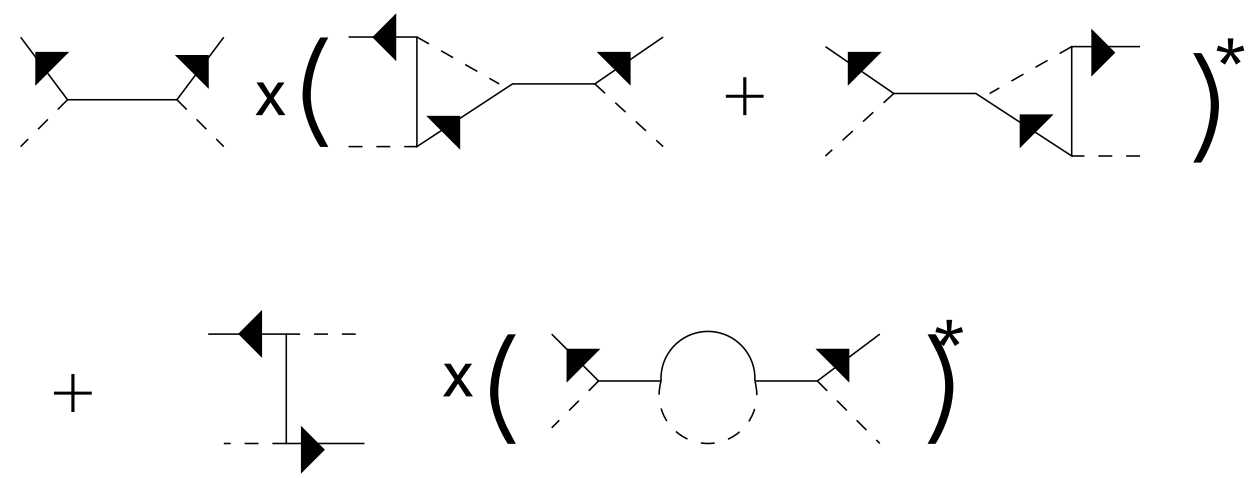

Figure 4: Interference terms contributing to $\sigma\left(\ell^{c} H^{*} \rightarrow \ell H\right)$ and involving the one loop vertex contributions as well as the term necessary to enforce the cancellation of the CP asymmetry.

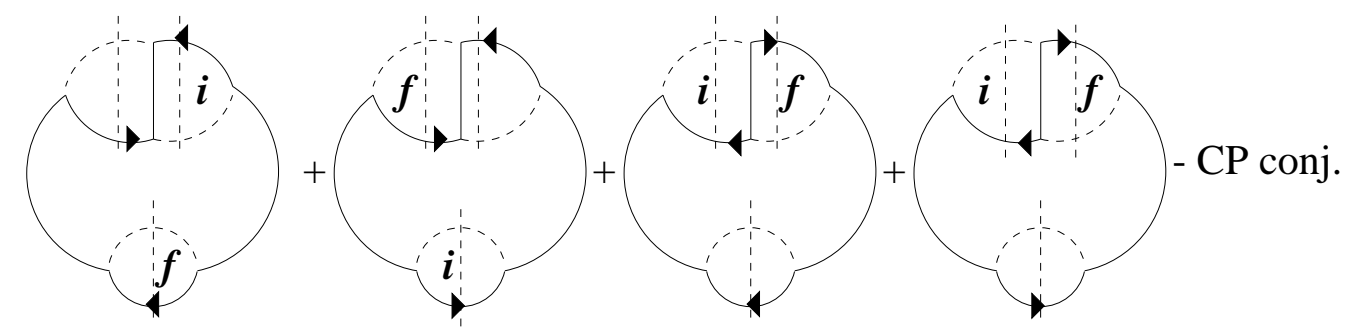

Figure 5: Pictorial representation of contribution to $\epsilon_{\sigma}$ from the diagrams in Figure 4.

The only remaining diagrams to be considered are the interference of the one-loop vertex diagrams with the tree level $u$-channel. Pictorially, they are represented in figure 6 , and they again cancel since the two diagrams change into each other under CP-conjugation.

To further interpret these results, consider for simplicity the case of two generations, and just the terms in the interference part of the cross sections involving the exchange of two $N_{1}$ and one $N_{2}$. First, for the interference contributions resulting from the self-energies diagrams depicted in figure 2, there will be three terms corresponding to:

i) the interference of the $N_{1}$ tree exchange with the $N_{1} \times N_{2}$ mixing.

ii) the interference of the $N_{1}$ tree exchange with the $N_{2} \times N_{1}$ mixing.

iii) the interference of the $N_{2}$ tree exchange with the $N_{1} \times N_{1}$ mixing.

If we consider the resonant production, i.e. with $s \simeq M_{1}^{2}$, the contribution 
in $i$ ) will factorise into the production of a real $N_{1}$ and its decay through mixing with $N_{2}$ (see ref. [14]). Similarly, ii) will correspond to the production of a real $N_{1}$ through mixing with $N_{2}$ and its subsequent decay. However, iii) does not factorise, since $N_{2}$ is off-shell, but as shown above its presence is crucial to cancel the $\mathrm{CP}$ asymmetry in the process.

The same classification applies to the contributions in figure 4, and there will be real $N_{1}$ production with $\mathrm{CP}$ violating decay; $\mathrm{CP}$ violating inverse decay producing a real $N_{1}$ which then decays (these first two contributions involving the vertex corrections); and then a non-factorisable term coming from the $N_{2} u$-channel exchange interfering with a self-energy diagram with two $N_{1}$ s.

From the previous discussion it is clear that no contribution to the cross section CP asymmetry (11) should survive, neither from the diagrams involving self-energies nor from those involving vertices. These vanishing results do not however forbid the possibility of obtaining a net cosmological lepton asymmetry: The crucial point is to take into account the expansion of the Universe, following, by the Boltzmann equation, the evolution of the heavy neutrinos and of the lepton number distributions. In this way, the different processes just discussed ( $i$ to iii), will take place at different times, and the expansion of the Universe will affect the external conditions at production and decay so as to make the cancellation among the different pieces no longer complete (see below). Here is where the departure from equilibrium becomes essential, since in equilibrium no asymmetry can be produced just due to the unitarity constraint 15, 16.

Let us consider in slightly more detail the evolution of the lepton number density. The off-shell contribution from CP violating $2 \rightarrow 2$ scatterings (iii above) can be included in the Boltzmann equation exploiting the constraint

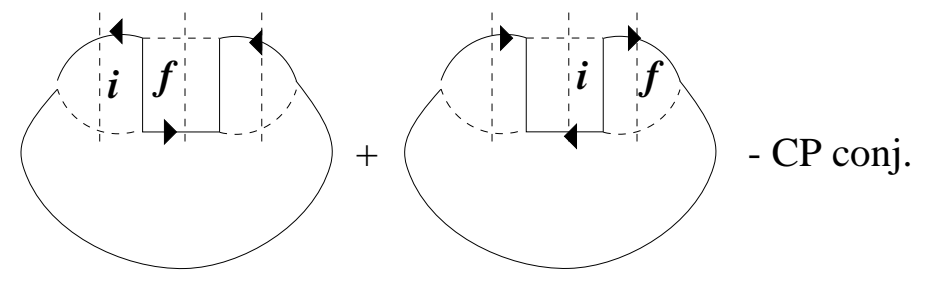

Figure 6: Pictorial representation of the contribution to $\epsilon_{\sigma}$ from the interference of the $u$-channel tree diagram with the one loop vertex corrections. 
from eq. (3), that is subtracting from the total cross section asymmetry (which is zero) the asymmetry associated with real intermediate states $(i$ and $i i$ above) [17]. This implies that the asymmetry produced in conditions of thermal equilibrium by inverse decays $(i i)$ and scatterings processes $(i i i)$ is cancelled by the asymmetry produced by direct decays]. But the first asymmetry can be erased, at least partially, by L violating scatterings (not necessarily $\mathrm{CP}$ violating) before the actual decays of the states $N_{1}$ take place. It is important here that the real intermediate states actually live for a long time by cosmological standards. As a consequence, between the production vertex and the decay one the temperature changes sufficiently as to allow first the compensation of the initial L asymmetry by L violating scatterings, which then by the time of the decay had already gone out of equilibrium. In this way, a non-vanishing asymmetry can eventually be generated.

This scenario requires a CP violating asymmetry in the decay of the $N_{1}$, which at lowest order is provided by the interference of the tree level diagrams with one loop diagrams that have non-zero absorptive parts. Hence, in a Boltzmann equation treatment of the $N_{i}$ distributions, both contributions of the vertex and of the self-energy diagrams should be included.

It is a pleasure to thank F. Botella, E.A. Paschos, N. Rius, A. Santamaria and A.Yu. Smirnov for useful discussions. This work was partially supported by CICYT, Spain, under grant No. AEN-96/1718.

\footnotetext{
${ }^{3}$ In the case of large hierarchies in the spectrum of the heavy states, the only particle relevant for the generation of the lepton asymmetry is typically the lightest singlet neutrino $N_{1}$. In the general case, however, an interplay between all processes takes place, and the sign of the lepton number asymmetry generated by any of the individual process $i, i i$ and iii depends on the heavy state considered.
} 


\section{References}

[1] M. Fukugita and T. Yanagida, Phys. Lett. B174 (1986) 45.

[2] A. Yu. Ignatiev, V. A. Kuzmin and M. E. Shaposhnikov, JETP Lett. 30 (1979) 688.

[3] F. J. Botella and J. Roldan, Phys. Rev. D44 (1991) 966.

[4] J. Liu and G. Segrè, Phys. Rev. D48 (1993) 4609.

[5] J. Liu and G. Segrè, Phys. Rev. D49 (1994) 1342.

[6] M. Flanz, E. A. Paschos and U. Sarkar, Phys. Lett. B345 (1995) 24; ibidem, B 384 (1996) 487 (E).

[7] L. Covi, E. Roulet and F. Vissani, Phys. Lett. B384 (1996) 169.

[8] L. Covi and E. Roulet, Phys. Lett. B399 (1997) 113.

[9] A. Pilaftsis, hep-ph/9707235.

[10] M. A. Luty, Phys. Rev. D45 (1992) 455.

[11] B. A. Campbell, S. Davidson and K. A. Olive, Nucl. Phys. B399 (1993) 111.

[12] M. Plümacher, Z. Phys. C 74 (1997) 549.

[13] M. Plümacher, hep-ph/9704231.

[14] W. Buchmüller and M. Plümacher, preprint DESY 97-190, hepph/9710460.

[15] S. Weinberg, Phys. Rev. Lett. 42 (1979) 850.

[16] E. W. Kolb and S. Wolfram, Nucl. Phys. B172 (1980) 224; ibidem, B195 (1982) 542 (E).

[17] See the detailed discussion in Sect. 2.3.2 of [16] as well as [10] and [12]. 